\title{
ON INTERSECTIONS OF COMPACTA IN EUCLIDEAN SPACE
}

\author{
A. N. DRANISHNIKOV
}

(Communicated by James E. West)

\begin{abstract}
Let $\mathbf{X}$ be a codimension-three tame compactum in Euclidean space $\mathbf{E}^{n}$. If $\operatorname{dim} \mathbf{X} \times \mathbf{Y}<n$, then every map $f: \mathbf{Y} \rightarrow \mathbf{E}^{n}$ can be approximated by map $g$ with $\mathbf{X} \cap \operatorname{Im} g=\varnothing$.
\end{abstract}

\section{INTRODUCTION}

This paper is a sequel to the recent series of works [M-Ru1-2], [K-L], [K], [Sp1-2] [D-S], [D-R-S1-2], [D2].

Suppose a compactum $\mathbf{X}$ lies in Euclidean space $\mathbf{E}^{n}$. Under what conditions can every map $f: \mathbf{Y} \rightarrow \mathbf{E}^{n}$ of compactum $\mathbf{Y}$ be approximated by a map whose image does not intersect $\mathbf{X}$ ? This paper suggests a solution of the problem in terms of the dimension of the product. Denote by $\mathbf{C}(\mathbf{X}, \mathbf{Z})$ the space of all continuous maps of $\mathbf{X}$ into $\mathbf{Z}$.

Theorem 1. Let $\mathbf{X}$ be a codimension-three tame compactum in Euclidean space $\mathbf{E}^{n}$. Suppose that $\operatorname{dim}(\mathbf{X} \times \mathbf{Y})<n$ for some compactum $\mathbf{Y}$. Then the space $\mathbf{C}\left(\mathbf{Y}, \mathbf{E}^{n}-\mathbf{X}\right)$ is dense in $\mathbf{C}\left(\mathbf{Y}, \mathbf{E}^{n}\right)$.

By the tameness of $\mathbf{X}$ we understand the equality $\operatorname{dem} \mathbf{X}=\operatorname{dim} \mathbf{X}$, where dem is Shtanko's embedding dimension [Sh], [E].

This theorem with the additional restriction $\operatorname{dim} \mathbf{X}+\operatorname{dim} \mathbf{Y} \leq n$ was proved in [D-Š] and [D-R-Šs]. The restriction $\operatorname{dim} \mathbf{X}+\operatorname{dim} \mathbf{Y} \leq n$ is very strong because some recent results in dimension theory [D1] imply that there exist compacta $\mathbf{X}$ and $\mathbf{Y}$ with $\operatorname{dim}(\mathbf{X} \times \mathbf{Y})<n$ and $\operatorname{dim} \mathbf{X}+\operatorname{dim} \mathbf{Y}=m$ for given $m<2 n-3$ (of course, $m>\max \{\operatorname{dim} \mathbf{X}, \operatorname{dim} \mathbf{Y}\}$ ).

Corollary 1 [D2]. Let $\mathbf{X}$ and $\mathbf{Y}$ be compacta with $\operatorname{dim} \mathbf{X}+\operatorname{dim} \mathbf{Y} \leq 4 / 3 n-1$. Suppose that $\operatorname{dim}(\mathbf{X} \times \mathbf{Y})<n$. Then every pair of maps $f: \mathbf{X} \rightarrow \mathbf{E}^{n}$ and $j: \mathbf{Y} \rightarrow \mathbf{E}^{n}$ has unstable intersection.

A pair of maps $f: \mathbf{X} \rightarrow \mathbf{Z}$ and $g: \mathbf{Y} \rightarrow \mathbf{Z}$ of compacta $\mathbf{X}$ and $\mathbf{Y}$ into a metric space $\mathbf{Z}$ is said to have an unstable intersection [D-R-Š2] if, for every

Received by the editors October 12, 1989 and, in revised form, March 12, 1990.

1980 Mathematics Subject Classification (1985 Revision). Primary 54C25, 55M10.

Key words and phrases. Compactum, cohomological dimension, unstable intersection. 
$\varepsilon>0$, there exists a pair of maps $f^{\prime}: \mathbf{X} \rightarrow \mathbf{Z}$ and $g^{\prime}: \mathbf{Y} \rightarrow \mathbf{Z}$ satisfying the conditions that $d\left(f, f^{\prime}\right)<\varepsilon$ and $d\left(g, g^{\prime}\right)<\varepsilon$ and with the property $\operatorname{Im} f^{\prime} \cap \operatorname{Im} g^{\prime}=\varnothing$.

Corollary 1 with the restriction $\operatorname{dim} \mathbf{X}+\operatorname{dim} \mathbf{Y} \leq n$ was proved in [D-R-Š2] and [Sp2]. It was also proved without this restriction in recent work [Sp-T].

Corollary 2 [D2]. Let $\mathbf{X}$ and $\mathbf{Y}$ be compacta with $\operatorname{dim}(\mathbf{X} \times \mathbf{Y})<n$. Then there exists a number $m$ such that every pair of maps $f: \mathbf{X} \rightarrow \mathbf{E}^{n+m}$ and $g: \mathbf{Y} \times \mathbf{I}^{m} \rightarrow$ $\mathbf{E}^{n+m}$ has an unstable intersection.

Here $\mathbf{I}^{m}$ is an $m$-dimensional cube.

Conjecture. The inequality $\operatorname{dim} \mathbf{X} \times \mathbf{Y}<n$ for a pair of compacta implies the instability of intersections for every pair of maps $f: \mathbf{X} \rightarrow \mathbf{E}^{n}$ and $g: \mathbf{Y} \rightarrow \mathbf{E}^{n}$.

The positive answer to the questions in any of the following problems implies the conjecture.

Problem 1. Let $\mathbf{X}$ and $\mathbf{Y}$ be compacta with $\operatorname{dim}(\mathbf{X} \times \mathbf{Y})<n$. Can a given map $f: \mathbf{X} \rightarrow \mathbf{E}^{n}$ be approximated by $f^{\prime}$ with the property $\operatorname{dim}\left(\operatorname{Im} f^{\prime} \times \mathbf{Y}\right)<n$ ?

Problem 2. Let $\mathbf{X}$ be a compactum with $\operatorname{dim} \mathbf{X} \leq n-2$, and let $G$ be an Abelian group. Can an arbitrary map $f: \mathbf{X} \rightarrow \mathbf{E}^{n}$ be approximated by $f^{\prime}$ with the property $c-\operatorname{dim}_{G} \operatorname{Im} f^{\prime} \leq c-\operatorname{dim}_{G} X$ ?

Recall that the cohomological dimension of a compactum $\mathbf{Z}$ with respect to $G$ as coefficient, $c-\operatorname{dim}_{G} \mathbf{Z}$, is the largest number $m$ for which there exists a closed subset $A \subset Z$ with $\check{H}^{m}(Z, A ; G) \neq 0$, where $\check{H}^{*}$ is a Čech cohomology group [Ku].

Problem 3. Let $\mathbf{X}$ and $\mathbf{Y}$ be compacta. Suppose that a pair of maps $f: \mathbf{X} \rightarrow \mathbf{E}^{n}$ and $g: \mathbf{Y} \rightarrow \mathbf{E}^{n}$ has a stable intersection. Does it imply that the pair $f: \mathbf{X} \rightarrow$ $\mathbf{E}^{n} \times\{0\} \hookrightarrow \mathbf{E}^{n+1}$ and $g \times \mathrm{id}_{I}: \mathbf{Y} \times I \rightarrow \mathbf{E}^{n+1}$, where $I=[-1,1]$, has a stable intersection in $\mathbf{E}^{n+1}$ ?

As a consequence of the proof of Theorem 1, a generalization of the Alexandrov theorem [A] for an arbitrary group is obtained.

\section{THE MAIN LEMMA}

A homology group $H_{k}(\mathbf{X} ; \mathbf{Z})$ with the integers as coefficients is denoted by $H_{k}(\mathbf{X})$.

Lemma 1. Let $\mathbf{M}$ be a simply connected $C W$-complex and $\mathbf{Y}$ be a compactum. If $c-\operatorname{dim}_{H_{k}(\mathbf{M})} \mathbf{Y} \leq k$ for all $k$, then $c-\operatorname{dim}_{\pi_{k}(\mathbf{M})} \mathbf{Y} \leq k$ for every $k$.

We will denote the homotopy group $\pi_{k}(\mathbf{M})$ by $\pi_{k}$ and the homology group $H_{k}(\mathbf{M})$ by $H_{k}$.

Let us consider a Postnikov tower for the space $\mathbf{M}$ [Spa]: $* \leftarrow E_{2} \leftarrow E_{3} \leftarrow$ $\cdots \leftarrow E_{n} \leftarrow \cdots$. 
Assertion 1. For each $n$ and arbitrary $\mathbf{G}$ there exist the equalities $H_{n}\left(\mathbf{E}_{n} ; \mathbf{G}\right)=$ $H_{n}\left(\mathbf{E}_{n+1} ; \mathbf{G}\right)=\ldots=H_{n}(\mathbf{M} ; \mathbf{G})$.

Proof. It is easy to see that $\mathbf{E}_{n}$ is homotopy equivalent to $C W$-complex $\mathbf{T}$, which is obtained from $\mathbf{M}$ by attaching cells of dimension $\geq n+2$. Hence $H_{n}\left(\mathbf{E}_{n} ; \mathbf{G}\right)=H_{n}(\mathbf{T} ; \mathbf{G})=H_{n}\left(\mathbf{T}^{(n+1)} ; \mathbf{G}\right)=H_{n}\left(\mathbf{M}^{(n+1)} ; \mathbf{G}\right)=H_{n}(\mathbf{M} ; \mathbf{G})$. The other equalities can be obtained similarly.

Recall that $\mathbf{Z}=\mathbf{Z} / p \mathbf{Z}$ and $\mathbf{Z}_{p^{\infty}}=\underline{\lim } \mathbf{Z}_{p^{k}}$.

Assertion 2. Let $\pi$ be an Abelian group with no element of order $p$, and suppose that each element of $\pi$ is divisible by $p$. Then $\widetilde{H}_{*}(K(\pi, n) ; \mathbf{G})=0$ for all $n$ if $\mathbf{G}=\mathbf{Z}_{p}$ or $\mathbf{G}=\mathbf{Z}_{p^{\infty}}$.

Here $K(\pi, n)$ is Eilenberg-McLane complex.

Proof. It is easy to verify that $\pi \otimes \mathbf{Z}\left[\frac{1}{p}\right]=\pi$. Let $\mathbf{X}_{[p]}$ denote a localization of space $\mathbf{X}$ out of the prime number $p$. Then $[\mathrm{Su}] K(\pi, n)_{[p]}=K\left(\pi_{[p]}, n\right)=$ $K\left(\pi \otimes \mathbf{Z}\left[\frac{1}{p}\right], n\right)=K(\pi, n)$. Hence

$$
H_{m}(K(\pi, n))=H_{m}\left(K(\pi, n)_{[p]}\right)=H_{m}(K(\pi, n)) \otimes \mathbf{Z}\left[\frac{1}{p}\right] .
$$

According to the universal coefficients formula we have $0 \rightarrow H_{m}(K(\pi, n)) \otimes$ $\mathbf{Z}\left[\frac{1}{p}\right] \otimes \mathbf{G} \rightarrow H_{m}(K(\pi, n) ; \mathbf{G}) \rightarrow\left(H_{m-1}(K(\pi, n)) \otimes \mathbf{Z}\left[\frac{1}{p}\right]\right) * \mathbf{G} \rightarrow 0$. In the case $G=\mathbf{Z}_{p}$ or $G=\mathbf{Z}_{p}$, both the right and the left sides of the sequence are zero. Assertion 3. Suppose that $H_{k}$ has no p-torsion and each element of $H_{k}$ is divisible by $p$ for all $k<n$. Then $\widetilde{H}_{*}\left(\mathbf{E}_{k} ; \mathbf{G}\right)=0$ for all $k<n$ if $\mathbf{G}=\mathbf{Z}_{p}$ or $\mathbf{G}=\mathbf{Z}_{p^{\infty}}$.

Proof. We will use induction on $k$.

If $k=1$, it is true by virtue of $\mathbf{E}_{1}=*$. Assume that the assertion is proved for some $k<n$. Let us consider the spectral sequence of the fibration $\mathbf{E}_{k+1} \rightarrow \mathbf{E}_{k}$ in the Postnikov tower:

$$
\mathbf{E}_{m, l}^{2}=H_{m}\left(\mathbf{E}_{k} ; H_{l}\left(K\left(\pi_{k+1}, k+1\right) ; \mathbf{G}\right)\right) .
$$

The universal coefficient formula gives us the sequence

$$
\begin{aligned}
0 & \rightarrow H_{m}\left(\mathbf{E}_{k}\right) \otimes H_{l}\left(K\left(\pi_{k+1}, k+1\right) ; \mathbf{G}\right) \rightarrow \mathbf{E}_{m, l}^{2} \\
& \rightarrow H_{m-1}\left(\mathbf{E}_{k}\right) * H_{l}\left(K\left(\pi_{k+1}, k+1\right) ; \mathbf{G}\right) \rightarrow 0 .
\end{aligned}
$$

Assertion 2 implies that $\mathbf{E}_{m, l}^{2}=0$ if $l>0$. If $l=0$ and $m>0$ then $\mathbf{E}_{m, l}^{2}=0$ by the assumption of induction. Since $\mathbf{E}_{m, l}^{2}=0$ if $(m, l) \neq(0,0)$ then $\widetilde{H}_{*}\left(\mathbf{E}_{k+1} ; \mathbf{G}\right)=0$.

Assertion 4. Let $f: \mathbf{E} \rightarrow \mathbf{B}$ be a fibration whose fibre $\mathbf{F}$ is $(n-1)$-connected. Suppose that $\widetilde{H}_{*}(\mathbf{B} ; \mathbf{G})=0$. Then the inclusion $\mathbf{F} \hookrightarrow \mathbf{E}$ induces an isomorphism $H_{n}(\mathbf{F} ; \mathbf{G}) \rightarrow H_{n}(\mathbf{E} ; \mathbf{G})$.

Proof. Consider the spectral sequence of $f$. 
Assertion 5. Let $f: \mathbf{E} \rightarrow \mathbf{B}$ be a fibration with (n-1)-connected fibre $\mathbf{F}$. Suppose that $\widetilde{H}_{*}\left(\mathbf{B} ; \mathbf{Z}_{p^{\infty}}\right)=0$. Then the inclusion $j: \mathbf{F} \hookrightarrow \mathbf{E}$ induces an isomorphism $j_{*}: H_{n+1}\left(\mathbf{F} ; \mathbf{Z}_{p^{\infty}}\right) \rightarrow H_{n+1}\left(\mathbf{E} ; \mathbf{Z}_{p^{\infty}}\right)$.

Proof. Consider the spectral sequence of the fibration $f$. It is enough to show that $\mathbf{E}_{1, n}^{2}=\mathbf{E}_{2, n}^{2}=0$.

Indeed $\mathbf{E}_{m, n}^{2}=H_{m}\left(\mathbf{B} ; H_{n}(\mathbf{F}) ; \mathbf{Z}_{p^{\infty}}\right)=H_{m}\left(\mathbf{B} ; H_{n}(\mathbf{F}) \otimes \mathbf{Z}_{p^{\infty}}\right)=0$. The last equality follows from the universal coefficients formula and stipulation

$$
\widetilde{H}^{*}\left(\mathbf{B} ; \mathbf{Z}_{p^{\infty}}\right)=0 .
$$

Denote by $\mathscr{G}$ Bokshtein's family of groups. It consists of the rationals $\mathbf{Q}, \mathbf{Z}_{p}=\mathbf{Z} / p \mathbf{Z}, p$-primary quasi-cyclic groups $\mathbf{Z}_{p^{\infty}}$, and localizations $\mathbf{Z}_{(p)}$ of the integers in $p$ where $p$ runs on all prime numbers.

Following Bokshtein, define a set $\mathscr{G}(G)$ for an arbitrary Abelian group $G$ :

(1) $\mathbf{Q} \in \mathscr{G}(\mathbf{G}) \Leftrightarrow \mathbf{Q} \otimes \mathbf{G} \neq 0$,

(2) $\mathbf{Z}_{(p)} \in \mathscr{G}(\mathbf{G}) \Leftrightarrow \mathbf{Z}_{p^{\infty}} \otimes \mathbf{G} \neq 0$,

(3) $\mathbf{Z}_{p} \in \mathscr{G}(\mathbf{G}) \Leftrightarrow \mathbf{G} \otimes \mathbf{Z}_{p^{\infty}}=0$ and $\mathbf{G} \otimes \mathbf{Z}_{p} \neq 0$,

(4) $\mathbf{Z}_{p^{\infty}} \in \mathscr{G}(\mathbf{G}) \Leftrightarrow \mathbf{Z}_{p^{\infty}} \otimes \mathbf{G}=0, \mathbf{Z}_{p} \otimes \mathbf{G}=0$, and $\mathbf{Z}_{p} * \mathbf{G}=0$.

Bokshtein's Theorem [Ku]. For arbitrary compactum $\mathbf{X}$ and arbitrary Abelian group $\mathbf{G}$ there is the equality

$$
c-\operatorname{dim}_{\mathbf{G}} \mathbf{X}=\max _{\mathbf{H} \in \mathscr{\mathscr { S }}(\mathbf{G})} c-\operatorname{dim}_{\mathbf{H}} \mathbf{X} .
$$

Proof of Lemma 1. Denote by $\delta_{k}$ the set $\left\{\mathbf{G}: c-\operatorname{dim}_{\mathbf{G}} \mathbf{Y} \leq k\right\}$. By the conditions of Lemma 1, we have $\mathbf{H}_{k} \in \delta_{k}$ and $\delta_{k} \subset \delta_{k+1}$ for all $k$. All we need to prove is the inclusion $\pi_{k} \in \delta_{k}$ for each $k$. We do it by induction on $k$.

Since $\pi_{1}=0$, then $\pi_{1} \in \delta_{1}$. Assume that, for $k<n$, inclusions $\pi_{k} \in \delta_{k}$ hold. We must prove the inclusion $\pi_{n} \in \delta_{n}$. By virtue of Bokshtein's Theorem,

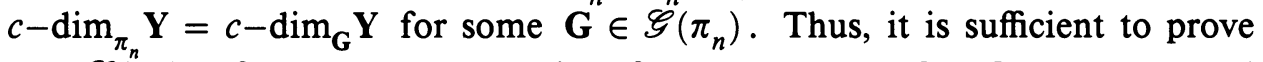
that $\mathscr{G}_{(}\left(\pi_{n}\right) \subset \delta_{n}$. We have to consider four cases: when $\mathbf{G}=\mathbf{Q}, \mathbf{Z}_{(p)}, \mathbf{Z}_{p}$, and $\mathbf{Z}_{p^{\infty}}$.

Suppose that $\mathbf{G} \in \mathscr{G}\left(\pi_{n}\right)$. Then, if $\mathbf{G} \in \delta_{n-1}$, it follows that (*) $\quad \mathbf{G} \in \delta_{n-1} \subset \delta_{n}$. Hence we can assume for each $\mathbf{G}$ that $\mathbf{G} \notin$ $\delta_{n-1}$.

Suppose that $\mathbf{G}=\mathbf{Q} \in \mathscr{G}\left(\pi_{n}\right)$. Then $(*)$ and the condition $\mathbf{H}_{k} \in \delta_{k}$ imply that $\cup_{i<n} \mathbf{H}_{i}$ has no element of infinite order. Let us consider a localization $\mathbf{M}_{(0)}$ of $\mathbf{M}$ in 0 . Then $\mathbf{H}_{k}\left(\mathbf{M}_{(0)}\right)=\mathbf{H}_{k} \otimes \mathbf{Q}=0$ for all $k<n$. Hurewicz's theorem implies $\mathbf{H}_{n}\left(\mathbf{M}_{(0)}\right)=\pi_{n}\left(\mathbf{M}_{(0)}\right)$. Since $\mathbf{H}_{n} \otimes \mathbf{Q}=\mathbf{H}_{n}\left(\mathbf{M}_{(0)}\right)=\pi_{n}\left(\mathbf{M}_{(0)}\right)=\pi_{n} \otimes \mathbf{Q} \neq$ 0 , the group $\mathbf{H}_{n}$ contains an element of infinite order. Hence $\mathbf{Q} \in \mathscr{G}\left(\mathbf{H}_{n}\right)$, by the definition of $\mathscr{G}\left(\mathbf{H}_{n}\right)$. Therefore, $\mathbf{Q} \in \delta_{n}$.

Suppose that $\mathbf{Z}_{(p)} \in \mathscr{G}\left(\pi_{n}\right)$. There are three possibilities: (1) $\mathbf{Q}$ and some $p$-torsion group are both $\in \delta_{n-1} ;(2) \mathbf{Q} \notin \delta_{n-1} ;$ (3) $\mathbf{Q} \in \delta_{n-1}$ and there is no $p$-torsion group in $\delta_{n-1}$. 
(1) From Bokshtein's inequality $[\mathrm{Ku}]$,

$$
c-\operatorname{dim}_{\mathbf{Z}_{(p)}} \mathbf{Y} \leq \max \left\{c-\operatorname{dim}_{\mathbf{Q}} \mathbf{Y}, \quad c-\operatorname{dim}_{\mathbf{Z}_{p} \infty} \mathbf{Y}+1\right\},
$$

it follows that $\mathbf{Z}_{(p)} \in \delta_{n}$.

(2) Consider a localization of $\mathbf{M}$ in prime $p$, and apply the above argument. By using modulo $p$-torsions and Hurewicz's theorem [Spa], we will obtain an equality $\mathbf{H}_{n} \otimes \mathbf{Z}_{(p)}=\pi_{n} \otimes \mathbf{Z}_{(p)}$. Since $\mathbf{Z}_{(p)} \otimes \mathbf{Z}_{p^{\infty}}=\mathbf{Z}_{p^{\infty}}$, this equality together with $\mathbf{Z}_{(p)} \in \mathscr{G}\left(\pi_{n}\right)$ implies $\mathbf{Z}_{(p)} \in \mathscr{G}\left(\mathbf{H}_{n}\right)$. Hence $\mathbf{Z}_{(p)} \in \delta_{n}$.

(3) In this case all elements of $\mathbf{H}_{i}$ for $i<n$ are divisible by $p$. Apply Assertions 3 and 4 to obtain $\mathbf{H}_{n}\left(\mathbf{E}_{n} ; \mathbf{Z}_{p^{\infty}}\right)=\mathbf{H}_{n}\left(\mathbf{K}\left(\pi_{n}, n\right) ; \mathbf{Z}_{p^{\infty}}\right)$. The universal coefficient formula implies that $\mathbf{H}_{n} \otimes \mathbf{Z}_{p^{\infty}}=\pi_{n} \otimes \mathbf{Z}_{p^{\infty}} \neq 0$. Therefore, $\mathbf{Z}_{(p)} \in \mathscr{G}\left(\mathbf{H}_{n}\right)$ and $\mathbf{Z}_{(p)} \in \delta_{n}$.

Suppose that $\mathbf{G}=\mathbf{Z}_{p} \in \mathscr{G}\left(\pi_{n}\right)$. There are two possibilities: (1) $\mathbf{Z}_{p^{\infty}} \in \delta_{n-1}$ and (2) $\mathbf{Z}_{p^{\infty}} \notin \delta_{n-1}$. In the first case, Bokshtein's inequality

$$
c-\operatorname{dim}_{\mathbf{Z}_{p}} \mathbf{Y} \leq c-\operatorname{dim}_{\mathbf{Z}_{p} \infty} \mathbf{Y}+1
$$

implies the inclusion $\mathbf{Z}_{p} \in \delta_{n}$. In the second case, due to Bokshtein's inequality

$$
c-\operatorname{dim}_{\mathbf{Z}_{p}} \mathbf{Y} \leq c-\operatorname{dim}_{\mathbf{Z}_{(p)}} \mathbf{Y},
$$

we can assume that $\mathbf{Z}_{(p)} \notin \delta_{n}$. Apply Assertions 1, 3, and 4 to obtain equalities $\mathbf{H}_{n}\left(\mathbf{M} ; \mathbf{Z}_{p}\right)=\mathbf{H}_{n}\left(\mathbf{E}_{n} ; \mathbf{Z}_{p}\right)=\mathbf{H}_{n}\left(\mathbf{K}\left(\pi_{n}, n\right) ; \mathbf{Z}_{p}\right)=\pi_{n} \otimes \mathbf{Z}_{p} \neq 0$. From the other side, $\mathbf{H}_{n}\left(\mathbf{M} ; \mathbf{Z}_{p}\right)=\mathbf{H}_{n}\left(\mathbf{E}_{n} ; \mathbf{Z}_{p}\right)=\mathbf{H}_{n} \otimes \mathbf{Z}_{p}$. Hence $\mathbf{H}_{n} \otimes \mathbf{Z}_{p} \neq 0$. Since $\mathbf{Z}_{(p)} \notin \delta_{n}$ and $\mathbf{H}_{n} \in \delta_{n}, \mathbf{Z}_{(p)} \notin \mathscr{G}\left(\mathbf{H}_{n}\right)$, and hence $\mathbf{H}_{n} \otimes \mathbf{Z}_{p^{\infty}}=0$. Then $\mathbf{Z}_{p} \in \mathscr{G}\left(\mathbf{H}_{n}\right) \subset \delta_{n}$.

Suppose at least that $\mathbf{G}=\mathbf{Z}_{p^{\infty}} \in \mathscr{G}\left(\pi_{n}\right)$. By virtue of Bokshtein's inequalities $[\mathrm{Ku}]$,

$$
c-\operatorname{dim}_{\mathbf{Z}_{p} \infty} \mathbf{Y} \leq c-\operatorname{dim}_{\mathbf{Z}_{p}} \mathbf{Y} \leq c-\operatorname{dim}_{\mathbf{Z}_{(p)}} \mathbf{Y}
$$

we can assume that $\mathbf{Z}_{p}, \mathbf{Z}_{(p)} \notin \delta_{n}$.

There are two cases: (1) $\mathbf{Q} \notin \delta_{n-1}$ and (2) $\mathbf{Q} \in \delta_{n-1}$. In the first case we consider the Serre class of Abelian groups $\mathbf{C}$ which consists of torsion groups having no element of order $p$ [Spa]. Hurewicz's modulo-C theorem implies a modulo-C equality $\mathbf{H}_{n}=\pi_{n}$. Hence $\mathbf{Z}_{p^{\infty}} \in \mathscr{G}\left(\mathbf{H}_{n}\right) \subset \delta_{n}$ by the definition of $\mathscr{G}\left(\mathbf{H}_{n}\right)$. In the second case, apply Assertions 3, 4, and 5 to obtain the isomorphism

$$
j_{*}: \mathbf{H}_{n+1}\left(\mathbf{K}(\pi, n) ; \mathbf{Z}_{p^{\infty}}\right) \rightarrow \mathbf{H}_{n+1}\left(\mathbf{E}_{n} ; \mathbf{Z}_{p^{\infty}}\right) \text {. }
$$

Since $\mathbf{H}_{n}\left(\mathbf{K}\left(\pi_{n}, n\right)\right) * \mathbf{Z}_{p^{\infty}}=\pi_{n} * \mathbf{Z}_{p^{\infty}} \neq 0$ we can assume that $\mathbf{H}_{n+1}\left(\mathbf{E}_{n} ; \mathbf{Z}_{p^{\infty}}\right) \neq$ 0 . The spectral sequence of the fibration $\mathbf{E}_{n+1} \rightarrow \mathbf{E}_{n}$ implies $\mathbf{H}_{n+1}\left(\mathbf{E}_{n+1} ; \mathbf{Z}_{p^{\infty}}\right)$ $\neq 0$. Therefore, $\mathbf{H}_{n+1} \otimes \mathbf{Z}_{p^{\infty}} \neq 0$ or $\mathbf{H}_{n} * \mathbf{Z}_{p^{\infty}} \neq 0$. The condition $\mathbf{H}_{n+1} \otimes$ $\mathbf{Z}_{p^{\infty}} \neq 0$ implies $\mathbf{Z}_{(p)} \in \mathscr{G}\left(\mathbf{H}_{n+1}\right) \subset \delta_{n+1}$. By virtue of Bokshtein's inequal- 
ity $c-\operatorname{dim}_{\mathbf{Z}_{p \infty}} \mathbf{Y} \leq \max \left\{c-\operatorname{dim}_{\mathbf{Q}} \mathbf{Y}, c-\operatorname{dim}_{\mathbf{Z}_{(p)}} \mathbf{Y}-1\right\}$, we can conclude that $\mathbf{Z}_{p^{\infty}} \in \delta_{n}$. The condition $\mathbf{H}_{n} * \mathbf{Z}_{p^{\infty}} \neq 0$ combined with the fact that $\mathbf{Z}_{p}$, $\mathbf{Z}_{(p)} \notin \mathscr{G}\left(\mathbf{H}_{n}\right)$ implies that $\mathbf{Z}_{p^{\infty}} \in \mathscr{G}\left(\mathbf{H}_{n}\right)$.

\section{Proof OF THEOREM 1}

Notation (Kuratowski). $\mathbf{X} \tau \mathbf{Z} \Leftrightarrow$ for every closed subset $\mathbf{A} \subset \mathbf{X}$ and every map $f: \mathbf{A} \rightarrow \mathbf{Z}$ there exists an extension $\bar{f}: \mathbf{X} \rightarrow \mathbf{Z}$ of $f$.

Recall that $\mathbf{X} \tau \mathbf{K}(\mathbf{G}, n)$ is equivalent to $c-\operatorname{dim}_{\mathbf{G}} \mathbf{X}<n[\mathrm{Ku}]$.

Assertion 6. Let $\mathbf{Y}$ and $\mathbf{Z}$ be homotopy-equivalent ANE-spaces. Then $\mathbf{X} \tau \mathbf{Y}$ if and only if $\mathbf{X} \tau \mathbf{Z}$.

Proof. Apply the homotopy extension theorem.

Assertion 7. Let $f: \mathbf{E} \rightarrow \mathbf{B}$ be a locally trivial fibration with fibre $\mathbf{F}$, and assume that $\mathbf{E}, \mathbf{F} \in A N E$ and that $\mathbf{B}$ is a polyhedron. Suppose that $\mathbf{X} \tau \mathbf{B}$ and $\mathbf{X} \tau \mathbf{F}$ for some compactum $\mathbf{X}$. Then $\mathbf{X} \tau \mathbf{E}$.

Proof. Consider a map $\varphi: \mathbf{A} \rightarrow \mathbf{E}$ where $\mathbf{A}$ is a closed subset of $\mathbf{X}$. By $\mathbf{X} \tau \mathbf{B}$ there exists an extension $\psi: \mathbf{X} \rightarrow \mathbf{B}$ of the map $f \circ \varphi$. For each point $x \in \mathbf{B}^{(0)}-f \varphi(\mathbf{A})$ choose a point $x^{\prime} \in f^{-1}(x)$. Here $\mathbf{B}^{(i)}$ denotes the $i$ dimensional skeleton of $\mathbf{B}$. By using $\mathbf{X} \tau \mathbf{F}$, extend the lifting of $\varphi$ to $\varphi^{\prime}$ : $\mathbf{A} \cup \psi^{-1}\left(\psi(\mathbf{A}) \cap \mathbf{B}^{(0)}\right) \rightarrow \mathbf{E}$. Define a map $\varphi_{0}: \psi^{-1}\left(\mathbf{B}^{(0)}\right) \cup \mathbf{A} \rightarrow \mathbf{E}$ by the formula

$$
\varphi_{0}(x)= \begin{cases}\varphi^{\prime}(x) & \text { if } x \in \psi^{-1}\left(\psi(\mathbf{A}) \cap \mathbf{B}^{(0)}\right) \cup \mathbf{A} \\ (\psi(x))^{\prime} & \text { if } x \in \psi^{-1}\left(\mathbf{B}^{(0)}-\psi(\mathbf{A})\right) .\end{cases}
$$

Assume that there is a map $\varphi_{k}: \psi^{-1}\left(\mathbf{B}^{(k)}\right) \cup \mathbf{A} \rightarrow \mathbf{E}$ such that $\left.\varphi_{k}\right|_{\mathbf{A}}=\varphi$ and $f \circ \varphi_{k}=\psi \mid \ldots$. Consider an arbitrary $(k+1)$-dimensional simplex $\sigma \subset$ $\mathbf{B}^{(k+1)}$. The local triviality of $f$ implies that there exists a fibre-preserving homeomorphism $h_{\sigma}: f^{-1}(\sigma) \rightarrow \sigma \times \mathbf{F}$. Let $\pi_{F}: \sigma \times \mathbf{F} \rightarrow \mathbf{F}$ be the projection. By virtue of $\mathbf{X} \tau \mathbf{F}$ there exists an extension $\eta_{\sigma}: \psi^{-1}(\sigma) \rightarrow \mathbf{B}$ of the map $\xi_{\sigma}=\pi_{F} \circ h_{\sigma} \circ \varphi_{k} \mid \ldots: \psi^{-1}\left(\sigma^{(k)}\right) \rightarrow \mathbf{B}$. The composition of diagonal product $\left(\left.\psi\right|_{\psi^{-1}(\sigma)}\right) \Delta \eta_{\sigma}$ and $h_{\sigma}^{-1}$ gives us a map $\beta_{\sigma}: \psi^{-1}(\sigma) \rightarrow f^{-1}(\sigma)$. The union of all $\beta_{\sigma}$ defines an extension $\varphi_{k+1}: \psi^{-1}\left(\mathbf{B}^{(k+1)}\right) \cup \mathbf{A} \rightarrow \mathbf{E}$ of $\varphi_{k}$. By virtue of the compactness of $\mathbf{X}$ there will be an inclusion $\mathbf{X} \subset \psi^{-1}\left(\mathbf{B}^{(k)}\right)$ for some $k$. In that case, $\varphi_{k}$ will be a required extension.

Lemma 2. Let $\mathbf{X}$ be a finite-dimensional compactum with the property

$$
c-\operatorname{dim}_{\pi_{k}(M)} \mathbf{X} \leq k
$$

for all $k$ where $\mathbf{M}$ is a simply connected $C W$-complex. Then $\mathbf{X} \tau \mathbf{M}$.

Proof. Let $m$ be the dimension of $\mathbf{X}$. Consider the Postnikov tower of space $\mathbf{M}: * \leftarrow \mathbf{E}_{2} \leftarrow \mathbf{E}_{3} \leftarrow \ldots \leftarrow \mathbf{E}_{m} \leftarrow \ldots$ Let $\mathbf{N}_{m}$ be a homotopy equivalent to 
$\mathbf{E}_{m} C W$-complex which is obtained by attaching to $\mathbf{M}$ cells of the dimensions $\geq m+2$. It is easy to see that $\mathbf{X} \tau \mathbf{N}_{m}$ implies $\mathbf{X} \tau \mathbf{M}$. By Assertion 6, $\mathbf{X} \tau \mathbf{E}_{m}$ implies $\mathbf{X} \tau \mathbf{N}_{m}$. Thus, it is sufficient to prove that $\mathbf{X} \tau \mathbf{E}_{m}$. Applying the Milnor construction of universal bundles $[\mathbf{M}]$, we can assume that the Postnikov tower consists of polyhedra with locally trivial fibrations. Apply Assertion 7 and use induction to conclude that $\mathbf{X} \tau \mathbf{E}_{m}$.

Lemmas 1, 2 imply the following theorem:

Theorem 2. For finite-dimensional compacta, an inequality $c-\operatorname{dim}_{\mathbf{G}} \mathbf{Y} \leq n$ implies the property $\mathbf{Y} \tau \mathbf{M}(\mathbf{G}, n)$, where $\mathbf{M}(\mathbf{G}, n)$ is a Moor space.

Remark. Since $\operatorname{dim} \mathbf{Y} \leq n \Leftrightarrow \mathbf{Y} \tau \mathbf{S}^{n}$, Theorem 2 is an extension for arbitrary $\mathbf{G}$ of the well-known Alexandroff theorem [A]: for finite-dimensional compacta the inequality $c-\operatorname{dim}_{\mathbf{Z}} \mathbf{Y} \leq n$ implies $\operatorname{dim} \mathbf{Y} \leq n$.

Proof of Theorem 1. Let $f: \mathbf{Y} \rightarrow \mathbf{E}^{n}$ be an arbitrary map and let $\varepsilon>0$ be given. We can approximate $f$ by $g$ with a polyhedron $\mathbf{K}$ as the image of $g$. Suppose that $\rho(f, g)<1 / 2 \varepsilon$. We assume that $\mathbf{K}$ is a subpolyhedron in $\mathbf{E}^{n}$ with respect to some triangulation $\tau$. Suppose that mesh $\tau<1 / 4 \varepsilon$. Consider the second barycentric subdivision $\beta^{2} \tau$ of $\tau$, and denote by $\mathbf{B}_{\sigma}$ the star of each simplex $\sigma \in \tau$ with respect to $\beta^{2} \tau$. Then for every pair $\sigma_{1}, \sigma_{2}$ we have $\mathbf{B}_{\sigma_{1} \cap \sigma_{2}}=\mathbf{B}_{\sigma_{1}} \cap \mathbf{B}_{\sigma_{2}}$. It is easy to see that $\mathbf{B}_{\sigma}$ is homeomorphic to the $n$-dimensional cell.

By $\mathbf{K}^{(i)}$ we denote the $i$-skeleton of $\mathbf{K}$ with respect to $\tau$. For every $\sigma \in$ $\mathbf{K}^{(0)}$, choose a point $x_{\sigma} \in \operatorname{Int} \mathbf{B}_{\sigma}-\mathbf{X}$. This defines a map $g_{0}: g^{-1}\left(\mathbf{K}^{(0)}\right) \rightarrow$ $\mathbf{E}^{n}-\mathbf{X}$. Assume that we can define a map $g_{i}: g^{-1}\left(\mathbf{K}^{(i)}\right) \rightarrow \mathbf{E}^{n}-\mathbf{X}$ such that, for every simplex $\sigma \subset \mathbf{K}^{(i)}$, there is the inclusion $g_{i}\left(g^{-1}(\sigma)\right) \subset \operatorname{Int} \mathbf{B}_{\sigma}$. Let $\sigma$ be an arbitrary $i+1$-dimensional simplex in $\mathbf{K}$. Denote by $\mathbf{M}$ the set Int $\mathbf{B}_{\sigma}-\mathbf{X}$. The equality $\operatorname{dem} \mathbf{X}=\operatorname{dim} \mathbf{X} \leq n-3$ implies that $\mathbf{M}$ is simply connected. Since $\operatorname{dim} \mathbf{X} \times \mathbf{Y}<n$, for any open subset $\mathbf{V} \subset \mathbf{Y}$, the equality $\mathbf{H}_{c}^{n}\left(\left(\operatorname{Int} \mathbf{B}_{\sigma} \cap \mathbf{X}\right) \times \mathbf{V} ; \mathbf{Z}\right)=0$ holds. By virtue of the Künnet formula, $\mathbf{H}_{c}^{k}\left(\mathbf{V} ; \mathbf{H}_{c}^{n-k}\left(\operatorname{Int} \mathbf{B}_{\sigma} \cap \mathbf{X}\right)\right)=0$ for $k \leq n$. Apply the Alexander duality to obtain $\mathbf{H}_{c}^{k}\left(\mathbf{V} ; \mathbf{H}_{k-1}(\mathbf{M})\right)=0$ for $k \leq n$. It means [Ku] that $c-\operatorname{dim}_{\mathbf{H}_{k}(\mathbf{M})} \mathbf{Y} \leq k$ for all $k$. Apply Lemmas 1 and 2 to obtain the property $\mathbf{Y} \tau \mathbf{M}$. Hence there exists a map $q_{\sigma}: g^{-1}(\sigma) \rightarrow \operatorname{Int} \mathbf{B}_{\sigma}-\mathbf{X}=\mathbf{M}$ such that the restriction of $q_{\sigma}$ onto $g^{-1}\left(\sigma^{(i)}\right)$ coincides with restriction $g_{i}$ onto $g^{-1}\left(\sigma^{(i)}\right)$. The union of $q_{\sigma}$ defines a map $g_{i+1}: g^{-1}\left(\mathbf{K}^{(i+1)}\right) \rightarrow \mathbf{E}^{n}-\mathbf{X}$ with the property $g_{i+1}\left(g^{-1}(\sigma)\right) \subset \operatorname{Int} \mathbf{B}_{\sigma}$ for each $\sigma \subset \mathbf{K}^{(i+1)}$. Note that $\mathbf{Y}=g_{n}^{-1}\left(\mathbf{K}^{(n)}\right)$. The map $g_{n}: \mathbf{Y} \rightarrow \mathbf{E}^{n}-\mathbf{X}$ has the property $\rho\left(g_{n}, f\right)<\varepsilon$.

\section{Proof of COROLlaries}

We called [D-R-Š2] a map $f: \mathbf{X} \rightarrow \mathbf{E}^{n}$ regular branched if for every $k \geq$ $0 \operatorname{dim} \mathbf{B}_{k}(f) \leq k \operatorname{dim} \mathbf{X}-(k-1) n$, where $\mathbf{B}_{k}(f)=\left\{z \in \mathbf{E}^{n}: \# f^{-1}(z) \geq k\right\}$. 
Denote by $\mathbf{R}\left(\mathbf{X}, \mathbf{E}^{n}\right)$ the set of all regular branched maps from $\mathbf{X}$ to $\mathbf{E}^{n}$. Theorem 3 [D-R-Š2, Theorem 3.1]. For every compactum $\mathbf{X}$, the set $\mathbf{R}\left(\mathbf{X}, \mathbf{E}^{n}\right)$ is of the second Baire category in $\mathbf{C}\left(\mathbf{X}, \mathbf{E}^{n}\right)$.

Lemma 3. Let $\mathbf{X}$ and $\mathbf{Y}$ be compacta such that $\operatorname{dim} \mathbf{X} \leq \operatorname{dim} \mathbf{Y}, \operatorname{dim}(\mathbf{X} \times \mathbf{Y})<n$ and $\operatorname{dim} \mathbf{X}+\operatorname{dim} \mathbf{Y} \leq(4 / 3) n-1$. Then $\mathbf{N}=\left\{f \in \mathbf{C}\left(\mathbf{X}, \mathbf{E}^{n}\right): \operatorname{dim}(\operatorname{Im} f \times \mathbf{Y})\right.$ $<n$ and $\operatorname{dim}(\operatorname{Im} f) \leq \operatorname{dim} \mathbf{X}\}$ is dense in $\mathbf{C}\left(\mathbf{X}, \mathbf{E}^{n}\right)$.

Proof. The set $\mathbf{N}_{2}=\left\{f \in \mathbf{C}\left(\mathbf{X}, \mathbf{E}^{n}\right): \operatorname{dim}(\operatorname{Im} f) \leq \operatorname{dim} \mathbf{X}\right\}$ is of the second Baire category. This fact is well known and follows, for example, from [D-RS2, Corollary 3.3]. It suffices to prove that $\mathbf{R}\left(\mathbf{X}, \mathbf{E}^{n}\right) \subset \mathbf{N}_{1}=\left\{f \in \mathbf{C}\left(\mathbf{X}, \mathbf{E}^{n}\right)\right.$ : $\operatorname{dim}(\operatorname{Im} f \times \mathbf{Y})<n\}$. Then $\mathbf{N}=\mathbf{N}_{1} \cap \mathbf{N}_{2}$ will be dense in $\mathbf{C}\left(\mathbf{X}, \mathbf{E}^{n}\right)$.

Since $\operatorname{dim} \mathbf{X}<\frac{2}{3} n$, it follows that $\mathbf{B}_{k}(f)=\varnothing$ for $k>2$.

Consider the map $f \times \operatorname{id}_{\mathbf{Y}}: \mathbf{X} \times \mathbf{Y} \rightarrow \operatorname{Im} f \times \mathbf{Y}$. Then, for every $k>2$, $\mathbf{B}_{k}\left(f \times \mathrm{id}_{\mathbf{Y}}\right)=\varnothing$. Since $\operatorname{dim} \mathbf{X} \leq \frac{2}{3} n-1 / 2, \operatorname{dim} \mathbf{B}_{2}\left(f \times \mathrm{id}_{\mathbf{Y}}\right)=\operatorname{dim}\left(\mathbf{B}_{2}(f) \times \mathbf{Y}\right) \leq$ $\operatorname{dim} \mathbf{B}_{2}(f)+\operatorname{dim} \mathbf{Y} \leq 2 \operatorname{dim} \mathbf{X}-n+\operatorname{dim} \mathbf{Y}=\operatorname{dim} \mathbf{X}+\operatorname{dim} \mathbf{Y}+\operatorname{dim} \mathbf{X}-n \leq$ $\frac{4}{3} n-1+\frac{2}{3} n-1 / 2-n=n-\frac{3}{2}$. Apply the Zarelua-Skordev two-to-one mapping theorem [Z], [Sk] to conclude that

$$
\operatorname{dim}(\operatorname{Im} f \times \mathbf{Y}) \leq \max \left\{\operatorname{dim}(\mathbf{X} \times \mathbf{Y}), \operatorname{dim} \mathbf{B}_{2}\left(f \times \operatorname{id}_{\mathbf{Y}}\right)+1\right\}<n .
$$

Proof of Corollary 1. Suppose that $\operatorname{dim} \mathbf{X}<\operatorname{dim} \mathbf{Y}$. According to Lemma 3, there exists a map $f^{\prime}: \mathbf{X} \rightarrow \mathbf{E}^{n} \quad 1 / 2 \varepsilon$-close to $f$ and with $\operatorname{dim}\left(\operatorname{Im} f^{\prime} \times \mathbf{Y}\right)<n$. Since $\operatorname{dim} \mathbf{X} \leq n-3$, the Shtanko theorem [Sh] implies that there exists a reembedding $\varphi: \operatorname{Im} f^{\prime} \rightarrow \mathbf{E}$ of the set $\operatorname{Im} f^{\prime}$ which is $\varepsilon / 2$-close to the initial embedding and has the tameness property. Theorem 1 applied to $\varphi\left(\operatorname{Im} f^{\prime}\right)$ and $\mathbf{Y}$ implies that there exists a map $g^{\prime}: \mathbf{Y} \rightarrow \mathbf{E}^{n}-\varphi\left(\operatorname{Im} f^{\prime}\right) \varepsilon$-close to a given map $g$. Thus, the maps $\varphi \circ f$ and $g^{\prime}$ are $\varepsilon$-close to $f$ and $g$, respectively, and have disjoint images.

Proof of Corollary 2. Choose a number $m$ so large that the inequality $\operatorname{dim} \mathbf{X}+$ $\operatorname{dim} \mathbf{Y}+m \leq \frac{4}{3}(n+m)-1$ holds, and then apply Corollary 1 .

\section{REFERENCES}

[A] P. Alexandroff, Dimensiotheorie ein Beitrag zur Geometrie der abgeschossenen Mengen, Math. Ann. 106 (1932), 161-238.

[D1] A. N. Dranishnikov, Homological dimension theory, Uspehi Mat. Nauk, 43 (1988), 11-55. (in Russian)

[D2] _ Spanier-Whitehead duality and stability of intersection of compacta, Trudy of Steklov Inst. (to appear). (Russian)

[D-R-Š1] A. N. Dranishnikov, D. Repovš, and E. V. Ščepin, A criterion for approximation of maps of 2-dimensional compacta into $R$ by embeddings, Abstracts Amer. Math. Soc. 10 (1989), No. 89 T-54-132.

[D-R-Š2] __, On intersection of compacta of complementary dimensions in Euclidean space, Topology Appl. (to appear).

[D-Š] A. N. Dranishnikov and E. V. Ščepin, On stability of intersections of compacta in Euclidean space, Uspekhi Mat. Nauk 44 (1989), 159-160. (Russian) 
[E] R. D. Edwards, Dimension theory, I, Lecture Notes in Math., vol. 438, Springer-Verlag, Berlin and New York, 1975, 195-211.

[K] J. Krasinkiewicz, Imbeddings into $R^{n}$ and dimension of products, Math. Inst. Polish Acad. Sci., preprint, Warsaw, 1988.

[K-L] J. Krasinkiewicz and K. Lorentz, Disjoint membranes in cubes, preprint, Warsaw 1987.

[Ku] V. I. Kuzminov, Homological dimension theory, Russian Math. Surveys 23 (1968), 1-45.

[M] J. Milnor, Construction of universal bundles, II, Ann. Math. 63 (1956), 430-436.

[M-Rui] D. McCuilough and L. R. Rubin, Intersections of separators and essential submanifolds of $I^{N}$, Fund. Math. 116 (1983), 131-142.

[Sh] M. A. Shtanko, Embeddings of compacta in Euclidean space, Mat. Sbornik 83 (1970), 234255. (Russian)

[Sk] G. Skordev, On dimension raising maps, Mat. Zametki 7 (1970), 697-705. (Russian)

[Spa] E. H. Spanier, Algebraic topology, McGraw-Hill, New York, 1966.

[Sp1] S. Spież, Imbeddings in $\mathbf{R}^{2 m}$ of m-dimensional compacta with $\operatorname{dim}(\mathbf{X} \times \mathbf{X})<2 m$, Math. Inst. Polish Acad. Sci., preprint, Warsaw, 1988.

[Sp2] _ On pairs of compacta with $\operatorname{dim}(\mathbf{X} \times \mathbf{Y})<\operatorname{dim} \mathbf{X}+\operatorname{dim} \mathbf{Y}$, preliminary report, Math. Inst. Polish Acad. Sci., Warsaw, 1989.

[Sp-T] S. Spież and H. Toruńczyk, Moving compacta in $R^{m}$ apart, preprint, Warsaw, 1989.

[Su] D. Sullivan, Geometric topology, part I: Localization, periodicity and Galois symmetry, MIT, Cambridge, MA, 1970.

[W] J. J. Walsh, Dimension, cohomological dimension, and cell-like mappings, Lecture Notes in Math., vol. 870, Springer-Verlag, Berlin and New York, 1981, pp. 105-118.

[Z] A. V. Zarelua, Finite-to-one maps of topological spaces and cohomological manifolds, Sibirskii Mat. Zurnal 10 (1969), 64-92. (Russian)

Department of Mathematics, University of Tennessee, KNoxville, Tennessee 37996-1301

Current address: Steklov Mathematical Institute, Moscow, USSR 117966 